\title{
Ketercapaian dan Keterkontrolan Sistem Deskriptor Diskrit Linier Positif
}

\author{
Yulia Retno Sari \\ Universitas Putra Indonesia "YPTK” Padang, Jalan Raya Lubuk Begalung, Padang \\ yuliaretnosari2012@gmail.com
}

DOI:https://doi.org/10.15642/mantik.2017.3.2.65-73

\begin{abstract}
Abstrak
Sistem deskriptor diskrit positif telah banyak digunakan dalam pemodelan bidang ekonomi, teknik, kimia dan sebagainya. Dalam penelitian ini dikaji tentang syarat perlu dan syarat cukup agar sistem deskriptor diskrit positif adalah tercapai positif dan terkontrol positif. Selain itu, juga dikaji tentang syarat perlu dan syarat cukup yang menjamin agar sistem diskrit $(\mathrm{E}, \mathrm{A}, \mathrm{B}) \geq 0$ terkontrol null.

Dengan metode aljabar linier dan Invers Drazin, dalam penelitian ini dibuktikan beberapa teorema agar sistem deskriptor diskrit $(E, A, B) \geq 0$ tercapai positif, terkontrol positif dan terkontrol null. Selain itu, diberikan contoh sebagai ilustrasi untuk memperkuat keberlakuan teorema yang telah dibuktikan.
\end{abstract}

Kata kunci : Invers drazin, sistem deskriptor diskrit positif, matriks non negatif, matriks nilpoten, sifat ketercapaian.

\begin{abstract}
A positive discrete descriptor system has been widely used in modeling economics, engineering, chemistry and others. In this research, we studied the necessary conditions and sufficient conditions for a positive discrete descriptors system is achieved positive and controlled postively. In addition, it is also studied on sufficient terms and conditions which ensure that discrete systems $(E, A, B) \geq 0$ are null controlled.

By using linear algebraic method and Inverse Drazin, this research has proved several theorems for discrete descriptors system $(\mathrm{E}, \mathrm{A}, \mathrm{B}) \geq 0$ achieved positive, controlled positively and controlled null. In addition, examples are given as illustrations to reinforce the validity of the proven theorems.
\end{abstract}

Key words : Inverse Drazin, discrete positive descriptor system, non negative matrix, nilpotent matrix, achieved properties.

\section{Pendahuluan}

Diberikan suatu sistem persamaan beda linier (linear difference equations) sebagai berikut :

$$
E \mathbf{x}(k+1)=A \mathbf{x}(k)+B \mathbf{u}(k), \quad k \in \mathbb{Z}_{+}
$$

dengan $E, A \in \mathbb{R}^{n \times n}$, dan $B \in \mathbb{R}^{n \times m}$. Dalam sistem (1), $\mathbf{x} \in \mathbb{R}^{n}$ menyatakan vektor state (keadaan) dan $\mathbf{u} \in \mathbb{R}^{\mathrm{m}}$ menyatakan vektor input (kontrol). Notasi $\mathbb{R}^{n \times m}$ menyatakan himpunan matriks-matriks riil berukuran $n \times m, \quad \mathbb{R}^{n}$ menyatakan himpunan vektor berdimensi $n$ dan
$\mathbb{Z}_{+}$menyatakan himpunan bilangan bulat non negatif. Dalam [5], sistem (1) dikatakan sebagai sistem deskriptor diskrit.

Jika $E$ adalah matriks non singular, maka solusi dari sistem (1) adalah

$$
\begin{aligned}
\mathbf{x}(k)=\left(E^{-1} A\right)^{k} \mathbf{x}(0)+ & \\
& \sum_{i=0}^{k-1}\left(E^{-1} A\right)^{k-i-1}\left(E^{-1} B\right) \mathbf{u}(i)
\end{aligned}
$$

Untuk $E$ singular, sistem (1) mungkin tidak mempunyai solusi. Hal ini disebabkan adanya 


\section{JURNAL MATEMATIKA "MANTIK" \\ Edisi: Oktober 2017. Vol. 03 No. 02}

kondisi awal yang tidak dapat memberikan solusi untuk sistem (1). Kondisi awal yang dapat memberikan solusi untuk sistem (1) disebut sebagai kondisi awal yang konsisten.

Dalam [9] dinyatakan bahwa sistem (1) mempunyai solusi tunggal jika untuk suatu kondisi awal yang konsisten $\mathbf{x}(0)$ berlaku $\operatorname{det}(\lambda E-A) \neq 0$ untuk suatu $\lambda \in \mathbb{C}$. Jika kondisi ini terpenuhi, maka solusi sistem (1) diberikan sebagai berikut:

$$
\begin{array}{r}
\mathbf{x}(k)=\left(\bar{E}^{D} \bar{A}\right)^{k} \bar{E}^{D} \bar{E} \mathbf{x}(0)+\sum_{i=0}^{k-1} \bar{E}^{D}\left(\bar{E}^{D} \bar{A}\right)^{k-i-1} \bar{B} \mathbf{u}(i) \\
-\left(I-\bar{E} \bar{E}^{D}\right) \sum_{i=0}^{q-1}\left(\bar{E}^{D} \bar{A}\right)^{i} \bar{A}^{D} \bar{B} \mathbf{u}(k+i)
\end{array}
$$

Dengan $\quad \bar{E}=(\lambda E-A)^{-1} E, \bar{A}=(\lambda E-A)^{-1} A, \bar{B}$ $=(\lambda E-A)^{-1} B$ dan $q$ adalah indeks dari matriks $\bar{E}$. Dalam hal $\operatorname{det}(\lambda E-A) \neq 0$ untuk suatu $\lambda \in \mathbb{C}$, sistem (1) disebut sebagai sistem deskriptor diskrit regular.

Perlu diperhatikan bahwa solusi $\mathbf{x}(k)$ untuk sistem (1) dapat bernilai negatif ataupun non negatif. Solusi $\mathbf{x}(k)$ dikatakan non negatif jika $\mathbf{x}(k) \geq 0$ dan dikatakan negatif jika $\mathbf{x}(k)<0$. Jika solusi $\mathbf{x}(k)$ untuk sistem (1) adalah non negatif, maka sistem (1) dikatakan sistem deskriptor diskrit positif [3]. Untuk selanjutnya sistem deskriptor diskrit positif dapat ditulis dengan sistem diskrit $(E, A, B) \geq$ 0 .

Salah satu isu penting tentang sistem deskriptor diskrit positif adalah masalah ketercapaian positif dan keterkontrolan positif. [3] telah mendefinisikan tentang ketercapaian positif dan keterkontrolan positif sistem diskrit $(E, A, B) \geq 0$. Untuk sistem diskrit $(E, A, B) \geq$ 0 , suatu keadaan $\mathbf{w} \in \mathbb{R}_{+}^{n}$ dikatakan tercapai positif jika terdapat $k \in \mathbb{Z}_{+}$dan suatu barisan kontrol $\mathbf{u}(j) \geq 0, j=0,1, \ldots, k+q-1$, yang membawa keadaan $\mathbf{x}(0)=\mathbf{0}$ kepada keadaan w pada waktu $k$. Sistem diskrit $(E, A, B) \geq$ 0 dikatakan tercapai positif jika untuk setiap $\mathbf{w} \in \mathbb{R}_{+}^{n}$ adalah tercapai positif.

Selain itu, sistem diskrit $(E, A, B) \geq 0$ dikatakan terkontrol positif jika untuk sebarang $\mathbf{x}_{\mathbf{0}}, \mathbf{x}_{\mathbf{f}} \in \mathbb{R}_{+}^{n}$, terdapat $k \in \mathbb{Z}_{+}$dan suatu barisan kontrol $\mathbf{u}(j) \geq 0, j=0,1, \ldots, k+q-1$, yang membawa keadaan $\mathbf{x}(0)=\mathbf{x}_{\mathbf{0}}$ kepada keadaan $\mathbf{x}(k)=\mathbf{x}_{\mathbf{f}}$. Untuk selanjutnya, sistem diskrit $(E, A, B) \geq 0$ yang tercapai positif disebut tercapai dan yang terkontrol positif disebut terkontrol. Penelitian ini membicarakan syarat perlu dan cukup untuk ketercapaian dan keterkontrolan sistem deskriptor diskrit linier positif.

Berdasarkan uraian dari latar belakang, maka yang menjadi permasalahan dalam penelitian ini adalah :

1. Syarat apakah yang harus dipenuhi oleh sistem diskrit $(E, A, B) \geq 0$ agar tercapai.

2. Syarat apakah yang harus dipenuhi oleh sistem diskrit $(E, A, B) \geq 0$ agar terkontrol.

Kajian penelitian ini bertujuan untuk membuktikan syarat yang menjamin agar sistem diskrit $(E, A, B) \geq 0$ adalah tercapai dan terkontrol.

Penelitian ini diharapkan dapat memperluas wawasan penulis serta pembaca pada umumnya dan diharapkan dapat memberikan konstribusi kepada pembaca agar lebih memahami pembuktian tentang ketercapaian dan keterkontrolan untuk sistem deskriptor diskrit linier positif.

\section{Kajian Teori \\ 2.1 Teori Matriks}

Matriks didefinisikan sebagai susunan bilangan-bilangan di dalam baris dan kolom yang membentuk jajaran empat persegi panjang [1].

Suatu matriks $A=\left[a_{i j}\right]_{i, j=1}^{n} \in \mathbb{R}^{n \times n}, \quad A$ dikatakan non negatif dinotasikan $A \geq 0$, jika $a_{i j} \geq 0, \forall i, j=1,2, \cdots, n$ dan $A$ dikatakan positif, dinotasikan $A>0$, jika $a_{i j}>0, \forall i, j=$ $1,2, \cdots, n$. Suatu vektor $\mathbf{x} \in \mathbb{R}^{n}$ dikatakan non negatif jika setiap komponennya non negatif, yakni $x_{i} \geq 0, i=1, \ldots, n$. Jika $\mathbf{x}$ non negatif maka ditulis $\mathbf{x} \geq 0$ atau $\mathbf{x} \in \mathbb{R}_{+}^{n}$, dengan $\mathbb{R}_{+}^{n}$ menyatakan himpunan $\mathbb{R}^{n}$ yang setiap komponennya adalah non negatif. Untuk vektor $\mathbf{x}$ yang positif dapat didefinisikan dengan cara yang sama.

Definisi 2.1. Matriks persegi A disebut matriks nilpoten jika $A^{n}=0$ dan $A^{n-1} \neq 0$ dengan $n \in$ $\mathbb{Z}_{+}$terkecil. Bilangan tersebut didefinisikan sebagai indeks nilpotensi dari matriks A. 
Dalam [1] dinyatakan bahwa jika A dan B adalah matriks-matriks sedemikian sehingga $A B=B A$, maka $\mathrm{A}$ dan $\mathrm{B}$ didefinisikan sebagai dua matriks yang komutatif.

Definisi 2.2 Misalkan $E, A \in \mathbb{R}^{m x n}$. Pasangan matriks $(E, A)$ dikatakan regular jika $m=n$ dan $\operatorname{det}(\lambda E-A) \neq 0$ untuk suatu $\lambda \in \mathbb{C}$. Jika berlaku sebaliknya maka pasangan matriks $(E, A)$ dikatakan non regular.

Definisi 2.3 Dimensi ruang baris atau ruang kolom matriks A disebut rank dari A dan ditulis $\operatorname{rank}(A)$.

Teorema 2.4 Misalkan terdapat matriks $A \in$ $\mathbb{R}^{m x n}$. Maka dimensi kernel (ruang penyelesaian dari $A x=0$ ) adalah

$$
n-\operatorname{rank}(A) \text {. }
$$

Definisi 2.5 Misalkan terdapat matriks $A \in$ $\mathbb{R}^{m x n}$. Maka image A disimbolkan dengan $\operatorname{Im}(A)$, didefinisikan sebagai ruang peta dari $\mathrm{A}$ yaitu

$$
\operatorname{Im}(A)=\left\{\boldsymbol{w} \in \mathbb{R}^{m} \mid \exists \boldsymbol{x} \in \mathbb{R}^{n} \ni \boldsymbol{w}=A \boldsymbol{x}\right\} .
$$

Definisi 2.6 Misalkan terdapat matriks $A \in$ $\mathbb{R}^{n x n}$, suatu vektor $x \in \mathbb{R}^{n}, x \neq 0$ dikatakan vektor eigen (eigenvector) dari A jika $A \boldsymbol{x}$ adalah kelipatan skalar dari $\mathbf{x}$, yakni

$$
A x=\lambda \boldsymbol{x}
$$

untuk suatu skalar $\lambda$. Skalar $\lambda$ dinamakan nilai eigen (eigenvalue) dari A.

Nilai $\lambda$ pada (3) merupakan akar dari polinomial karakteristik :

$$
\operatorname{det}(\lambda I-A)=0 \text {. }
$$

Teorema Cayley-Hamilton [14] menyatakan bahwa jika polinomial karakteristik dari matriks A adalah

$$
p(\lambda)=a_{0}+a_{1} \lambda+a_{2} \lambda^{2}+\cdots+a_{n-1} \lambda^{n-1}+\lambda^{n},
$$

maka

$$
p(A)=a_{0} I+a_{1} A+a_{2} A^{2}+\cdots+a_{n-1} A^{n-1}+A^{n}=0
$$

Berikut ini akan disajikan beberapa hal penting mengenai invers Drazin dari suatu matriks $A_{n x n}$ yang diambil dari [9]. Invers
Drazin berguna untuk mencari solusi sistem deskriptor diskrit.

Definisi 2.7 Misalkan $A \in \mathbb{R}^{n x n}$. Indeks dari matriks $\mathrm{A}$, ditulis ind $(A)$, didefinisikan sebagai bilangan bulat non negatif terkecil q sedemikian sehingga

$$
\operatorname{rank}\left(A^{q}\right)=\operatorname{rank}\left(A^{q+1}\right) .
$$

Definisi 2.8 Misalkan $A \in \mathbb{R}^{n x n}$. Invers Drazin dari A, ditulis $A^{D}$, adalah suatu matriks yang memenuhi tiga syarat berikut :

1. $A A^{D}=A^{D} A$,

2. $A^{D} A A^{D}=A^{D}$,

3. $A^{D} A^{q+1}=A^{q}$, dimana q merupakan indeks dari A.

Invers Drazin $A^{D}$ dari suatu matriks persegi A selalu ada dan tunggal [4]. Jika A adalah matriks non singular, maka invers klasik $A^{-1}$ memenuhi sifat invers Drazin seperti yang diberikan dalam definisi (2.8). Dalam hal ini $A^{D}=A^{-1}$.

Berikut ini akan dipaparkan proses untuk menentukan invers Drazin dari suatu matriks persegi. Misalkan $A \in \mathbb{R}^{n x n}$ mempunyai nilai eigen nol dengan multiplisitas aljabar 1 dan nilai eigen berbeda $\lambda_{i}$ dengan multiplisitas aljabar $n_{i}, i=1,2, \ldots, r$. Jika $m=n_{1}+n_{2}+$ $\cdots+n_{r}$, maka $m+1=n$. Berdasarkan Teorema Cayley-Hamilton, invers Drazin $A^{D}$ dapat ditulis sebagai polinomial dalam A.

Perhatikan polinomial berikut :

$$
p(\lambda)=\lambda^{l}\left(a_{0}+a_{1} \lambda+\cdots+a_{m-1} \lambda^{m-1}\right)
$$

Koefisien $a_{0}, a_{1}, \ldots, a_{m-1}$ pada (4) dapat ditentukan dengan menyelesaikan sistem persamaan berikut :

$$
\begin{gathered}
\frac{1}{\lambda_{i}} p\left(\lambda_{i}\right) \\
\frac{-1}{\lambda_{i}^{2}}=p^{\prime}\left(\lambda_{i}\right) \\
\vdots \\
\frac{(-1)^{n_{i}-1}\left(n_{i}-1\right) !}{\left(\lambda_{i}\right)^{n_{i}}}=p^{\left(n_{i}-1\right)}\left(\lambda_{i}\right), \text { untuk } i=1,2, \ldots, r
\end{gathered}
$$

Lema berikut dapat digunakan untuk menghitung invers Drazin dari suatu matriks persegi. 
Lema 2.9 Jika $p(\lambda)$ didefinisikan oleh (4) dan (5), maka :

$$
A^{D}=p(A) .
$$

Sebagai ilustrasi dari Lema 2.9, perhatikan contoh berikut :

$$
A=\left[\begin{array}{ccrc}
2 & 4 & 6 & 5 \\
1 & 4 & 5 & 4 \\
0 & -1 & -1 & 0 \\
-1 & -2 & -3 & -3
\end{array}\right]
$$

Nilai eigen dari A adalah $0,0,1$ dan 1 . Untuk matriks A diatas,

$$
p(\lambda)=\lambda^{2}\left(a_{0}+a_{1} \lambda\right)
$$

Dengan menggunakan (5), diperoleh :

$$
1=a_{0}+a_{1},-1=2 a_{0}+3 a_{1} .
$$

Solusi dari (8) adalah $a_{0}=4$ dan $a_{1}=-3$. Jadi, berdasarkan Lema 2.9,

$$
\begin{aligned}
A^{D} & =A^{2}(4 I-3 A) \\
& =\left[\begin{array}{cccc}
3 & 8 & 11 & 11 \\
2 & 7 & 9 & 9 \\
-1 & -3 & -4 & -4 \\
-1 & -3 & -4 & -4
\end{array}\right]\left[\begin{array}{llll}
4 & 0 & 0 & 0 \\
0 & 4 & 0 & 0 \\
0 & 0 & 4 & 0 \\
0 & 0 & 0 & 4
\end{array}\right] \\
& -\left[\begin{array}{cccc}
6 & 12 & 18 & 15 \\
3 & 12 & 15 & 12 \\
0 & -3 & -3 & 0 \\
-3 & -6 & -9 & -9
\end{array}\right] \\
& =\left[\begin{array}{cccc}
3 & -1 & 2 & 2 \\
2 & 1 & 3 & 3 \\
-1 & 0 & -1 & -1 \\
-1 & 0 & -1 & -1
\end{array}\right]
\end{aligned}
$$

Lema 2.10 Misalkan $A, B \in \mathbb{C}^{n x n}$, 1. Jika $A B=B A$, maka

$$
\begin{aligned}
A B^{D} & =B^{D} A, \\
B A^{D} & =A^{D} B, \\
A^{D} B^{D} & =B^{D} A^{D} .
\end{aligned}
$$

2. Jika $A B=B A$ dan $\operatorname{ker} A \cap \operatorname{ker} B=\{\boldsymbol{0}\}$, maka

$$
\left(I-A A^{D}\right) B B^{D}=1-A A^{D} .
$$

\subsection{Ruang Vektor}

Pada bagian ini akan dibicarakan konsep vektor yang digunakan pada pembahasan.

Definisi 2.11 Misalkan $\mathbf{v}_{\mathbf{1}}, \mathbf{v}_{\mathbf{2}}, \ldots, \mathbf{v}_{\mathbf{n}}$ adalah vektor dan $r_{1}, r_{2}, \ldots, r_{n}$ skalar maka vektor,

$$
w=r_{1} \mathbf{v}_{\mathbf{1}}+r_{2} \mathbf{v}_{\mathbf{2}}+\cdots+r_{n} \mathbf{v}_{\mathbf{n}}
$$

adalah kombinasi linier dari $\mathbf{v}_{\mathbf{1}}, \mathbf{v}_{\mathbf{2}}, \ldots, \mathbf{v}_{\mathbf{n}}$. Himpunan semua kombinasi linier dari $\mathbf{v}_{\mathbf{1}}, \mathbf{v}_{\mathbf{2}}, \ldots, \mathbf{v}_{\mathbf{n}}$ dikatakan membangun $\mathbf{v}_{\mathbf{1}}, \mathbf{v}_{\mathbf{2}}, \ldots, \mathbf{v}_{\mathbf{n}}$ dan dinotasikan sebagai $\operatorname{span}\left\{\mathbf{v}_{\mathbf{1}}, \mathbf{v}_{\mathbf{2}}, \ldots, \mathbf{v}_{\mathbf{n}}\right\}$.

$\operatorname{Span}\left\{\mathbf{v}_{\mathbf{1}}, \mathbf{v}_{\mathbf{2}}, \ldots, \mathbf{v}_{\mathbf{n}}\right\}$

$=\left\{r_{1} \mathbf{v}_{\mathbf{1}}+r_{2} \mathbf{v}_{\mathbf{2}}+\cdots+r_{n} \mathbf{v}_{\mathbf{n}} \mid r_{1}, r_{2}, \ldots, r_{n}\right.$ adalah skalar $\}$.

Definisi 2.12 Misalkan vektor $\mathbf{v}_{\mathbf{1}}, \mathbf{v}_{\mathbf{2}}, \ldots, \mathbf{v}_{\mathbf{n}}$ dikatakan bebas linier jika $r_{1}, r_{2}, \ldots, r_{n}$ adalah skalar dan

$$
r_{1} \mathbf{v}_{\mathbf{1}}+r_{2} \mathbf{v}_{\mathbf{2}}+\cdots+r_{n} \mathbf{v}_{\mathbf{n}}=0
$$

hanya dipenuhi oleh $r_{1}=0, r_{2}=0, \ldots, r_{n}=0$.

Definisi 2.13 Ruang vektor $V$ dikatakan hasil tambah langsung dari subruang $W_{1}, W_{2}, \ldots, W_{n}$, jika

1. $V=\sum_{i=1}^{n} W_{i}$ dan

2. $W_{j} \cap\left(\sum_{\substack{i=1 \\ i \neq j}}^{n} W_{i}\right)=\{\mathbf{0}\}$; untuk semua $j=1,2, \ldots, n$.

Hasil tambah langsung ditulis dengan notasi,

$$
\begin{gathered}
V=W_{1} \underset{\substack{\text { atau } \\
n}}{W_{2} \oplus \ldots \oplus W_{n}} \\
V=\underset{i=1}{\bigoplus} W_{i} .
\end{gathered}
$$

\subsection{Proyeksi}

Definisi 2.14 Jika $T: V \rightarrow W$ adalah sebuah fungsi yang memetakan sebuah ruang vektor $\mathrm{V}$ kesebuah ruang vektor $\mathrm{W}$, maka $\mathrm{T}$ disebut sebagai transformasi linier (linier transformasi) dari V ke W, jika semua vektor $\mathbf{u}$ dan $\mathbf{w}$ pada $\mathrm{V}$ dan semua skalar c,

1. $T(\mathbf{u}+\mathbf{v})=T(\mathbf{u})+T(\mathbf{v})$.

2. $T(c \mathbf{u})=c T(\mathbf{u})$

Dalam kasus khusus dimana $V=W$, transformasi linier $T: V \rightarrow V$ disebut sebagai operator linier (linier operator) pada $\mathrm{V}$.

Teorema 2.15 Misalkan $G: F^{n} \rightarrow F^{m}$ adalah transformasi linier. Maka terdapat sebuah matriks A ukuran $m \times n$ sedemikian sehingga $G=T_{A}$.

Definisi 2.16 Misalkan $V=S \oplus S^{c}$ dengan $S^{c}$ adalah komplemen dari S. Pemetaan $P: V \rightarrow V$ yang didefinisikan sebagai

$$
P\left(\boldsymbol{s}+\boldsymbol{s}^{\boldsymbol{c}}\right)=\boldsymbol{s},
$$

dengan $\boldsymbol{s} \in S$ dan $\boldsymbol{s}^{\boldsymbol{c}} \in S^{c}$ disebut proyeksi pada $S$ sepanjang $S^{c}$. 
Teorema 2.17 Misalkan $P$ adalah suatu proyeksi pada $S$ sepanjang $S^{C}$ maka

1. $\operatorname{Im}(P)=S$ dan $\operatorname{ker}(P)=S^{c}$,

2. $V=\operatorname{Im}(P) \oplus \operatorname{ker}(P)$.

Teorema 2.18 Suatu operator linier $P$ adalah proyeksi jika dan hanya jika $P^{2}=P$.

\subsection{Solusi Sistem Deskriptor Diskrit}

Perhatikan kembali sistem deskriptor diskrit (1) dengan kondisi awal x(0). Jika entrientri dari A, B dan E tidak bergantung terhadap waktu, maka sistem pada persamaan (1) disebut time-invariant. Sebaliknya, jika entri-entri dari A, B dan E bergantung terhadap waktu, maka sistem (1) disebut time-varying. Asumsikan bahwa

$$
\operatorname{det}(\lambda E-A) \neq 0 \text {, untuk suatu } \lambda \in \mathbb{C} \text {. }
$$

Dengan mengalikan kedua ruas (1) dengan $(\lambda E-A)^{-1}$, diperoleh :

$$
\mathbf{x}(\mathrm{k}+1)=\overline{\mathrm{A}} \mathbf{x}(\mathrm{k})+\overline{\mathrm{B}} \mathbf{u}(\mathrm{k}),
$$

dengan

$$
\begin{aligned}
& \bar{E}=(\lambda E-A)^{-1} E, \bar{A}=(\lambda E-A)^{-1} A, \text { dan } \\
& \bar{B}=(\lambda E-A)^{-1} B .
\end{aligned}
$$

Lema 2.19 Untuk matriks $\bar{E}$ dan $\bar{A}$ yang didefinisikan dalam (14) berlaku,

1. $\bar{A} \bar{E}=\bar{E} \bar{A}$,

2. $\operatorname{ker}(\bar{A}) \cap \operatorname{ker}(\bar{E})=\{\mathbf{0}\}$

Bukti.

1. Berdasarkan persamaan (14), diperoleh

$$
\begin{aligned}
\lambda \bar{E}-\bar{A}= & \lambda(\lambda E-A)^{-1} E-(\lambda \\
& -A)^{-1} A \\
= & (\lambda E-A)^{-1}(\lambda E-A) \\
= & I,
\end{aligned}
$$

atau dapat ditulis

$$
\bar{A}=\lambda \bar{E}-I \text {. }
$$

Akibatnya,

$$
\bar{A} \bar{E}=(\lambda \bar{E}-I) \bar{E}=\bar{E}(\lambda \bar{E}-I)=\bar{E} \bar{A} .
$$

2. Misalkan bahwa $\mathbf{x} \in \operatorname{ker} \bar{A} \cap \operatorname{ker} \bar{E}$. Maka $\bar{A} \mathbf{x}=\mathbf{0}$ dan $\bar{E} \mathbf{x}=\mathbf{0}$, dan $(\lambda E-A) \mathbf{x}=\mathbf{0} . \quad$ Karena $\lambda \bar{E}-\bar{A}=I$, maka $\mathbf{x}=\mathbf{0}$.

Teorema 2.20 Solusi dari (13) dengan kondisi awal $\mathbf{x}(0)$ yang konsisten adalah
$\mathbf{x}(k)=\left(\bar{E}^{D} \bar{A}\right)^{k} \bar{E}^{D} \bar{E} \mathbf{x}(0)+$

$\sum_{i=0}^{k-1} \bar{E}^{D}\left(\bar{E}^{D} \bar{A}\right)^{k-i-1} \bar{B} \mathbf{u}(i)-$

$\left(-\bar{E} \bar{E}^{D}\right) \sum_{i=0}^{q-1}\left(\bar{E} \bar{A}^{D}\right)^{i} \bar{A}^{D} \bar{B} \mathbf{u}(k+1)$,

dengan $q$ adalah indeks dari matriks $\bar{E}$.

\section{Bukti.}

Misalkan $\bar{E}$ dan $\bar{A}$ didefinisikan seperti dalam (14). Berdasarkan Lema 2.19 (1) berlaku $\bar{A} \bar{E}=$ $\bar{E} \bar{A}$. Akibatnya, menurut Lema 2.10 (1) diperoleh $\bar{A} \bar{E}^{D}=\bar{E}^{D} \bar{A}$. Selanjutnya,

$$
\begin{aligned}
\bar{E} \mathbf{x}(k+1)=\left(\bar{E}^{D} \bar{A}\right)^{k+1} \bar{E} \mathbf{x}(0) & \\
& +\sum_{i=0}^{k}\left(\bar{E}^{D} \bar{A}\right)^{k-i} \bar{E} \bar{E}^{D} \bar{B} \mathbf{u}(i) \\
& -\left(I-\bar{E} \bar{E}^{D}\right) \sum_{i=0}^{q-1}\left(\bar{E} \bar{A}^{D}\right)^{i+1} \bar{B} \mathbf{u}(k \\
& +i+1)
\end{aligned}
$$

dan

$$
\begin{aligned}
\bar{A} \mathbf{x}(k)=\left(\bar{E}^{D} \bar{A}\right)^{k+1} \bar{E} \mathbf{x}(0)+\sum_{i=0}^{k-1}\left(\bar{E}^{D} \bar{A}\right)^{k-i} \bar{B} \mathbf{u}(i) \\
-\left(I-\bar{E} \bar{E}^{D}\right) \sum_{i=0}^{q-1}\left(\bar{E} \bar{A}^{D}\right)^{i+1} \bar{B} \mathbf{u}(k \\
+i)
\end{aligned}
$$

Berdasarkan Lema 2.19 berlaku $\bar{A} \bar{E}=\bar{E} \bar{A}$ dan $\operatorname{ker}(\bar{A}) \cap \operatorname{ker}(\bar{E})=\{\boldsymbol{0}\}, \quad$ akibatnya menurut Lema 2.10 (2) diperoleh $\left(I-\bar{E} \bar{E}^{D}\right) \bar{A} \bar{A}^{D}=I-$ $\bar{E} \bar{E}^{D}$, sehingga

$$
\begin{aligned}
& \left(I-\bar{E} \bar{E}^{D}\right)\left(\bar{E} \bar{A}^{D}\right)^{q} \\
& =\left(I-\bar{E} \bar{E}^{D}\right) \bar{A} \bar{A}^{D}\left(\bar{E} \bar{A}^{D}\right)^{q} \\
& =\left(I-\bar{E} \bar{E}^{D}\right) \bar{A} \bar{A}^{D} \bar{E}^{q}\left(\bar{A}^{D}\right)^{q} \\
& =\bar{A} \bar{A}^{D} \bar{E}^{q}\left(\bar{A}^{D}\right)^{q}-\bar{E} \bar{E}^{D} \bar{A} \bar{A}^{D} \bar{E}^{q}\left(\bar{A}^{D}\right)^{q} \\
& =\bar{A} \bar{A}^{D} \bar{E}^{q}\left(\bar{A}^{D}\right)^{q}-\bar{E} \bar{E}^{D} \bar{A} \bar{A}^{D} \bar{E}^{D} \bar{E}^{q+1}\left(\bar{A}^{D}\right)^{q} \\
& =\bar{A} \bar{A}^{D} \bar{E}^{q}\left(\bar{A}^{D}\right)^{q}-\bar{A} \bar{A}^{D} \bar{E}^{D} \bar{E}^{q+1}\left(\bar{A}^{D}\right)^{q} \\
& =\bar{A} \bar{A}^{D} \bar{E}^{q}\left(\bar{A}^{D}\right)^{q}-\bar{A} \bar{A}^{D} \bar{E}^{q}\left(\bar{A}^{D}\right)^{q} \\
& =0 .
\end{aligned}
$$

Selanjutnya, untuk membuktikan bahwa (15) merupakan solusi dari (13), akan ditunjukkan

$$
\begin{aligned}
& \bar{E} \mathbf{x}(k+1)-\bar{A} \mathbf{x}(k) \\
= & \bar{B} \mathbf{u}(k) \cdot \bar{E} \mathbf{x}(k+1)-\bar{A} \mathbf{x}(k) \\
= & \bar{E} \bar{E}^{D} \bar{B} \mathbf{u}(k)-(I- \\
& \left.\bar{E} \bar{E}^{D}\right) \sum_{i=0}^{q-1}\left[\left(\bar{E} \bar{A}^{D}\right)^{i+1} \bar{B} \mathbf{u}(k+i+1)+\right. \\
& \left.\left(\bar{E} \bar{A}^{D}\right)^{i} \bar{B} \mathbf{u}(k+i)\right] \\
= & \bar{E} \bar{E}^{D} \bar{B} \mathbf{u}(k)\left(I-\bar{E} \bar{E}^{D}\right) \sum_{i=0}^{q-1}\left[\left(\bar{E} \bar{A}^{D}\right)^{i} \bar{B} \mathbf{u}(k+\right. \\
& \left.i)-\left(\bar{E} \bar{A}^{D}\right)^{i+1} \bar{B} \mathbf{u}(k+i+1)\right]
\end{aligned}
$$




$$
\begin{aligned}
= & \bar{E} \bar{E}^{D} \bar{B} \mathbf{u}(k)+\left(I-\bar{E} \bar{E}^{D}\right)[(\bar{B} \mathbf{u}(k)- \\
& \left.\left(\bar{E} \bar{A}^{D}\right) \bar{B} \mathbf{u}(k+1)\right)+\left(\left(\bar{E} \bar{A}^{D}\right) \bar{B} \mathbf{u}(k+1)-\right. \\
& \left.\left(\bar{E} \bar{A}^{D}\right)^{2} \bar{B} \mathbf{u}(k+2)\right)+\cdots+ \\
& \left(\left(\bar{E} \bar{A}^{D}\right)^{q-2} \bar{B} \mathbf{u}(k+q-2)-\right. \\
& \left.\left(\bar{E} \bar{A}^{D}\right)^{q-1} \bar{B} \mathbf{u}(k+q-1)\right)+ \\
& \left(\left(\bar{E} \bar{A}^{D}\right)^{q-1} \bar{B} \mathbf{u}(k+q-1)-\right. \\
& \left.\left(\bar{E} \bar{A}^{D}\right)^{q} \bar{B} \mathbf{u}(k+q)\right) \\
= & \bar{E} \bar{E}^{D} \bar{B} \mathbf{u}(k)+\left(I-\bar{E} \bar{E}^{D}\right)[\bar{B} \mathbf{u}(k) \\
= & \left.\quad-\left(\bar{E} \bar{A}^{D}\right)^{q} \bar{B} \mathbf{u}(k+q)\right] \\
& \bar{E} \bar{E}^{D} \bar{B} \mathbf{u}(k)+\left(I-\bar{E} \bar{E}^{D}\right) \bar{B} \mathbf{u}(k) \\
& \quad-\left(I-\bar{E} \bar{E}^{D}\right)\left(\bar{E} \bar{A}^{D}\right)^{q} \bar{B} \mathbf{u}(k \\
= & \bar{E} \bar{E}^{D} \bar{B} \mathbf{u}(k)+\bar{B} \mathbf{u}(k)-\bar{E} \bar{E}^{D} \bar{B} \mathbf{u}(k) \\
= & \bar{B} \mathbf{u}(k) \quad
\end{aligned}
$$

Jadi, solusi (15) memenuhi sistem (13).

\subsection{Sistem Deskriptor Diskrit Positif}

Teorema 2.21 Untuk sistem deskriptor diskrit $(\mathrm{E}, \mathrm{A}, \mathrm{B})$, asumsikan bahwa $\mathrm{EE}^{\mathrm{D}} \geq 0, \mathrm{EA}=\mathrm{AE}$ dan $\operatorname{ker} E \cap \operatorname{ker} A=\{\mathbf{0}\} . \quad$ Sistem diskrit $(E, A, B) \geq 0$ jika dan hanya jika $E^{D} A \geq$ $0, E^{\mathrm{D}} \mathrm{B} \geq 0 \quad$ dan $\quad\left(\mathrm{I}-\mathrm{E}^{\mathrm{D}} \mathrm{E}\right)\left(\mathrm{E} A^{\mathrm{D}}\right)^{\mathrm{i}} \mathrm{A}^{\mathrm{D}} \mathrm{B} \leq 0$ dengan $\mathrm{q}$ adalah indeks dari $\mathrm{E}$.

\section{Bukti.}

$(\Rightarrow)$ Misalkan bahwa sistem diskrit $(E, A, B) \geq$ 0 . Karena $E A=A E$ dan $\operatorname{ker} E \cap \operatorname{ker} A=\{\mathbf{0}\}$, untuk setiap kondisi awal yang konsisten $\mathbf{x}(0) \geq \mathbf{0}$ dan untuk setiap kontrol non negatif, berlaku $\mathbf{x}(k) \geq \mathbf{0} \quad \forall k \in \mathbb{Z}_{+}$,

$$
\begin{aligned}
\mathbf{x}(k)=\left(E^{D} A\right)^{k} & E^{D} E \mathbf{x}(0) \\
& +\sum_{i=0}^{k-1} E^{D}\left(E^{D} A\right)^{k-i-1} B \mathbf{u}(i) \\
& -(I \\
& \left.-E E^{D}\right) \sum_{i=0}^{q-1}\left(E A^{D}\right)^{i} A^{D} B \mathbf{u}(k \\
& +i)
\end{aligned}
$$

Akan dibuktikan bahwa $E^{D} A \geq 0$. Selanjutnya, karena $E E^{D} \geq 0$, maka $\mathbf{x}(0)=E E^{D} \mathbf{e}_{i} \geq \mathbf{0}$, $i=1,2, \cdots, n$, merupakan kondisi awal yang konsisten, dengan $\mathbf{e}_{i} \in \mathbb{R}_{+}^{n}$ adalah vektor satuan ke- $i$. Dengan menggunakan kontrol $\mathbf{u}(j)=$ $\mathbf{0}, j=0,1, \cdots, k+q-1$, maka pada $k=1$, $\mathbf{x}(1)=\left(E^{D} A\right)^{1} E^{D} E \mathbf{x}(0)=E^{D} A E^{D} E E E^{D} \mathbf{e}_{i}$
Karena $E A=A E$, maka berdasarkan Lema 2.10 diperoleh $E^{D} A=A E^{D}$. Dengan menggunakan Definisi 2.8 yaitu $E^{D} E=E E^{D}$ dan $E^{D} E E^{D}=$ $E^{D}$, diperoleh :

$$
\begin{aligned}
\mathbf{x}(1) & =E^{D} A E E^{D} E E^{D} \mathbf{e}_{i} \\
& =E^{D} A E E^{D} \mathbf{e}_{i} \\
& =A E^{D} E E^{D} \mathbf{e}_{i} \\
& =A E^{D} \mathbf{e}_{i} .
\end{aligned}
$$

Karena $\mathbf{x}(1) \geq \mathbf{0}$ dan $\mathbf{e}_{i} \geq \mathbf{0}$ untuk setiap $i=$ $1,2, \cdots, n$, maka $E^{D} A \geq 0$. Berikutnya akan dibuktikan bahwa $E^{D} B \geq 0$. Ambil $\mathbf{x}(0)=\mathbf{0}$, $\mathbf{u}(j)=\mathbf{0}, j=1,2, \cdots, q$, dan $\mathbf{u}(0)=\mathbf{e}_{i} \in \mathbb{R}_{+}^{m}$ dengan $q$ adalah indeks dari $E$. Pada $k=1$,

$$
\mathbf{x}(1)=E^{D}\left(E^{D} A\right)^{0} B \mathbf{u}(0)=E^{D} B \boldsymbol{e}_{i} .
$$

Karena $\mathbf{x}(1) \geq \mathbf{0}$ dan $\mathbf{e}_{i} \geq \mathbf{0}$ untuk setiap $i=$ $1,2, \cdots, m$, maka $E^{D} B \geq 0$. Akhirnya, akan dibuktikan bahwa $\left(I-E^{D} E\right)\left(E A^{D}\right)^{i} A^{D} B \leq 0$ , $\forall i=0,1, \cdots, q-1$ dengan $q$ adalah indeks dari $E$. Dengan mengambil $\mathbf{x}(0)=\mathbf{0}, \mathbf{u}(k)=$ $\mathbf{e}_{i} \in \mathbb{R}_{+}^{m}, \quad$ dan $\quad \mathbf{u}(j)=\mathbf{0}, j \neq k, j=$ $0,1, \cdots, k+q-1$, diperoleh

$$
\mathbf{x}(k)=-\left(I-E^{D} E\right) A^{D} B \mathbf{e}_{i} .
$$

Karena $\mathbf{x}(k) \geq \mathbf{0}$ dan $\mathbf{e}_{i} \geq \mathbf{0}$ untuk setiap $i=$ $1,2, \cdots, m$, maka $\left(I-E^{D} E\right) A^{D} B \leq 0$. Dengan mengambil $\mathbf{u}(k+h)=\mathbf{e}_{i}$ untuk setiap $i=$ $1,2, \cdots, m$ dan $\mathbf{u}(j)=\mathbf{0}, j \neq k+h, \quad j=$ $0, \cdots, k+q-1$, maka diperoleh :

$$
\mathbf{x}(k)=-\left(I-E^{D} E\right)\left(E A^{D}\right)^{h} A^{D} B \boldsymbol{e}_{i} .
$$

Karena $\mathbf{x}(k) \geq \mathbf{0}$ dan $\mathbf{e}_{i} \geq \mathbf{0}$ untuk setiap $i=$ $1,2, \cdots, m$, maka

$$
\left(I-E^{D} E\right)\left(E A^{D}\right)^{h} A^{D} B \leq 0,
$$

untuk setiap $h=1,2, \ldots, q-1$.

$(\Leftarrow) \quad$ Misalkan $E^{D} A \geq 0, \quad E^{D} B \geq 0$ dan $\left(I-E^{D} E\right)\left(E A^{D}\right)^{i} A^{D} B \leq 0$, untuk setiap $i=$ $0,1, \cdots, q-1$ dengan $q$ adalah indeks dari $E$. Akibatnya, solusi dari sistem deskriptor diskrit $(E, A, B)$ adalah non negatif, $\mathbf{x}(k) \geq \mathbf{0}$, untuk setiap kontrol $\mathbf{u}(j) \geq \mathbf{0}, j=1,2, \cdots, k+q+1$, $k \in \mathbb{Z}_{+}$, sehingga sistem diskrit $(E, A, B) \geq 0$.

Teorema 2.22. Misalkan sistem diskrit $(\mathrm{E}, \mathrm{A}, \mathrm{B}) \geq 0$ dengan $\mathrm{EE}^{\mathrm{D}} \geq 0$ dan $\mathrm{EA}=\mathrm{AE}$. Sistem (E, A, B) adalah terkontrol null jika dan hanya jika $E^{\mathrm{D}} \mathrm{A}$ adalah suatu matriks nilpoten.

\section{Bukti.}

$(\Leftarrow)$ Misalkan $E^{D} A$ adalah suatu matriks nilpoten dengan indeks nilpotensi $l$. Akan dibuktikan bahwa sistem $(E, A, B)$ terkontrol null. Karena indeks nilpotensi dari matriks $E^{D} A$ adalah $l$, pilih $k \geq l$ dan barisan $\operatorname{kontrol} \mathbf{u}(j)=$ 
$\mathbf{0}, j=0,1, \ldots, k+q-1$, maka $\mathbf{x}(k)=\mathbf{0}$. Jadi, sistem $(E, A, B)$ adalah terkontrol null.

$(\Longrightarrow)$ Misalkan sistem diskrit $(E, A, B) \geq 0$ adalah terkontrol null maka untuk sebarang $\mathbf{x}_{\mathbf{0}} \in \mathbb{R}_{+}^{n}$ ada $k \in \mathbb{Z}_{+}$dan pilih barisan kontrol $\mathbf{u}(j)=\mathbf{0}, j=0,1, \ldots, k+q-1 \quad$ sedemikian sehingga

$$
\mathbf{0}=\left(E^{D} A\right)^{k} E^{D} E \mathbf{x}_{\mathbf{0}}
$$

Jika diambil $\mathbf{x}_{\mathbf{0}}=E^{D} E \mathbf{e}_{i}$ dengan $\mathbf{e}_{i}$ adalah vektor satuan ke-i, maka Persamaan 16 dapat ditulis menjadi

$$
\begin{aligned}
\left(E^{D} A\right)^{k} E^{D} E & E^{D} E \mathbf{e}_{i}=\left(E^{D} A\right)^{k} E^{D} E \mathbf{e}_{i} \\
& =\left(E^{D}\right)^{k} A^{k} E^{D} E \mathbf{e}_{i} \\
& =\left(E^{D}\right)^{k-1} E^{D} A^{k} E^{D} E \mathbf{e}_{i} \\
& =\left(E^{D}\right)^{k-1} A^{k} E^{D} E E^{D} \mathbf{e}_{i} \\
& =\left(E^{D}\right)^{k-1} A^{k} E^{D} \mathbf{e}_{i} \\
& =\left(E^{D}\right)^{k-1} E^{D} A^{k} \mathbf{e}_{i} \\
& =\left(E^{D}\right)^{k} A^{k} \mathbf{e}_{i} \\
& =\left(E^{D} A\right)^{k} \mathbf{e}_{i}=\mathbf{0} .
\end{aligned}
$$

untuk setiap $i=1,2, \ldots, n$. Karena $\left(E^{D} A\right)^{k} \mathbf{e}_{i}=$ 0 maka terdapat $l \in \mathbb{R}_{+}^{n}$ sedemikian sehingga $\left(E^{D} A\right)^{l}=0$, yaitu $E^{D} A$ adalah matriks nilpoten.

\section{Hasil dan Pembahasan}

Misalkan $\quad \mathcal{R}_{k}(E, A, B)$ menyatakan himpunan keadaan tercapai dalam waktu $k$, yaitu

$$
\mathcal{R}_{k}(E, A, B)=\{\mathbf{x}(k) \mid \mathbf{x}(k) \text { tercapai pada waktu } k\}
$$

Perhatikan bahwa, $\mathcal{R}_{k}(E, A, B)$ dibangun oleh kolom-kolom dari sub matriks non negatif berikut,

$$
\begin{aligned}
{\left[E^{D} B\left|E^{D} E^{D} A B\right|\right.} & \ldots\left|E^{D}\left(E^{D} A\right)^{k-1} B\right|-(I \\
& \left.-E^{D} E\right) A^{D} B|\ldots|-(I \\
& \left.\left.-E^{D} E\right)\left(E A^{D}\right)^{q-1} A^{D} B\right]
\end{aligned}
$$

Oleh karena itu, $\mathcal{R}_{k}(E, A, B)$ dapat juga ditulis sebagai berikut,

$\mathcal{R}_{k}(E, A, B)$

$=\left\langle E^{D} B, E^{D} E^{D} A B, \ldots, E^{D}\left(E^{D} A\right)^{k-1} B,-(I\right.$

$\left.\left.-E^{D} E\right) A^{D} B, \ldots,-\left(-E^{D} E\right)\left(E A^{D}\right)^{q-1} A^{D} B\right\rangle$

Selanjutnya, misalkan $\mathcal{R}_{\infty}(E, A, B)$ menyatakan himpunan keadaan tercapai dalam waktu hingga, maka

$$
\begin{aligned}
& \mathcal{R}_{\infty}(E, A, B) \\
& =\bigcup_{k=1}^{\infty} \mathcal{R}_{k}(E, A, B) \\
& =\{\mathbf{x} \mid \mathbf{x} \text { tercapai dalam waktu hingga }\} .
\end{aligned}
$$

Lema berikut memberikan syarat perlu dan cukup untuk ketercapaian sistem diskrit $(E, A, B) \geq 0$.

\section{Lema 3.1}

Sistem diskrit $(E, A, B) \geq$

0 adalah tercapai jika dan hanya jika

$$
\mathcal{R}_{\infty}(E, A, B)=\mathbb{R}_{+}^{n} \text {. }
$$

\section{Bukti}

$(\Rightarrow)$ Misalkan sistem diskrit $(E, A, B) \geq 0$ adalah tercapai. Akan dibuktikan bahwa $\mathcal{R}_{\infty}(E, A, B)=\mathbb{R}_{+}^{n} . \quad$ Ambil $\quad \mathbf{x}(j) \in$ $\mathcal{R}_{\infty}(E, A, B)$ maka $\mathbf{x}(j) \in \mathcal{R}_{j}(E, A, B)$. Ini bermakna bahwa $\mathbf{x}(j)$ tercapai dalam waktu $j$ sehingga $\mathbf{x}(j) \in \mathbb{R}_{+}^{n}$. Jadi $\mathcal{R}_{\infty}(E, A, B) \subseteq$ $\mathbb{R}_{+}^{n}$. Berikutnya, ambil sebarang $\mathbf{x}(j) \in \mathbb{R}_{+}^{n}$. Akan dibuktikan $\mathbf{x}(j) \in \mathcal{R}_{\infty}(E, A, B)$. Karena sistem diskrit $(E, A, B) \geq 0$, maka untuk setiap keadaan awal $\mathbf{x}_{\mathbf{0}} \in \mathbb{R}_{+}^{n}$, terdapat $\quad \mathbf{u}(j) \geq \mathbf{0}, j=0,1, \ldots, k+q-1$ sedemikian sehingga $\mathbf{x}(j) \geq \mathbf{0}, \forall k \in \mathbb{Z}_{+}$. Ini menunjukkan bahwa setiap $\mathbf{x}(j) \in \mathbb{R}_{+}^{n}$ tercapai dari keadaan $\mathbf{0}$. Sehingga $\mathbf{x}(j) \in$ $\mathcal{R}_{j}(E, A, B)$ untuk suatu $j$. Jadi $\mathbf{x}(j) \in$ $\mathcal{R}_{\infty}(E, A, B)$.

$(\Leftarrow)$ Misalkan $\mathcal{R}_{\infty}(E, A, B)=\mathbb{R}_{+}^{n}$, akan dibuktikan bahwa sistem diskrit $(E, A, B) \geq 0$ adalah tercapai. Telah diketahui

$$
\mathcal{R}_{\infty}(E, A, B)=\bigcup_{k=1}^{\infty} \mathcal{R}_{k}(E, A, B)
$$

mendefenisikan bahwa $\mathbf{x}$ tercapai dalam waktu hingga. Maka jelas, sistem diskrit $(E, A, B) \geq 0$ adalah tercapai.

Teorema 3.2 Sistem diskrit $(E, A, B) \geq$ 0 dengan $E E^{D} \geq 0$ dan $E A=$ $A E$ adalah tercapai jika dan hanya jika,

dan

$$
\mathcal{F}_{\infty}(E, A, B)=\operatorname{Im}\left(E E^{D}\right)
$$

$$
\mathcal{B}(E, A, B)=\operatorname{ker}\left(E E^{D}\right) .
$$




\section{Bukti}

Dari lema 3.1, diketahui bahwa sistem diskrit $(E, A, B) \geq 0$ tercapai jika dan hanya jika $\mathcal{R}_{\infty}(E, A, B)=\mathbb{R}_{+}^{n}$. Dari klaim diketahui bahwa

$$
\mathcal{R}_{k}(E, A, B)=F_{k}(E, A, B) \oplus \mathrm{B}(E, A, B)
$$
akibatnya

$$
\begin{aligned}
\mathbb{R}_{+}^{n} & =\mathcal{R}_{\infty}(E, A, B)=\bigcup_{k=1}^{\infty} \mathcal{R}_{k}(E, A, B) \\
= & \bigcup_{k=1}^{\infty} F_{k}(E, A, B) \oplus \mathrm{B}(E, A, B) \\
& =F_{\infty}(E, A, B) \oplus \mathrm{B}(E, A, B) \\
& =\operatorname{Im}\left(E E^{D}\right) \oplus \operatorname{ker}\left(E E^{D}\right)
\end{aligned}
$$

Sehingga sistem deskret $(E, A, B) \geq 0$ tercapai jika dan hanya jika

dan

$$
F_{\infty}(E, A, B)=\operatorname{Im}\left(E E^{D}\right)
$$

$$
\mathrm{B}(E, A, B)=\operatorname{ker}\left(E E^{D}\right) .
$$

Dari teorema 2.22 dan 3.1 dapat disimpulkan,

\section{Teorema 3.3}

Diberikan sistem diskrit $(E, A, B) \geq 0$ dengan $E E^{D} \geq 0$ dan $E A=A E$, maka sistem adalah terkontrol jika dan hanya jika $\mathcal{R}_{\infty}(\mathrm{E}, \mathrm{A}, \mathrm{B})=$ $\mathbb{R}_{+}^{\mathrm{n}}$ dan $\mathrm{E}^{\mathrm{D}} \mathrm{A}$ adalah matriks nilpoten.

\section{Bukti}

$(\Rightarrow)$ Misalkan sistem diskrit $(E, A, B) \geq 0$ dengan $E E^{D} \geq 0$ dan $E A=A E$ adalah terkontrol. Akan dibuktikan $\mathcal{R}_{\infty}(E, A, B)=\mathbb{R}_{+}^{n} \quad$ dan $\quad E^{D} A$ adalah matriks nilpoten. Karena sistem diskrit $(E, A, B) \geq 0$ terkontrol maka untuk setiap $\quad \mathbf{x}_{\mathbf{f}} \in \mathbb{R}_{+}^{n}$ tercapai dari sebarang keadaan awal $\mathbf{x}_{\mathbf{0}} \in \mathbb{R}_{+}^{n}$ khususnya $\mathbf{x}_{\mathbf{0}}=$ 0. Akibatnya sistem diskrit $(E, A, B) \geq 0$ adalah tercapai. Berdasarkan lema 3.1 maka

$$
\mathcal{R}_{\infty}(E, A, B)=\mathbb{R}_{+}^{n}
$$

Selanjutnya, keterkontrolan dari sistem diskrit $(E, A, B) \geq 0$ juga berakibat sistem diskrit $(E, A, B) \geq 0$ terkontrol null. Berdasarkan teorema 2.22, maka $E^{D} A$ adalah matriks nilpoten.
$(\Leftarrow)$ Misalkan $\mathcal{R}_{\infty}(E, A, B)=\mathbb{R}_{+}^{n}$ dan $E^{D} A$ adalah suatu matriks nilpoten dengan indeks nilpotensi $l$. Akan dibuktikan sistem diskrit $(E, A, B) \geq 0$ terkontrol. Ambil $\mathbf{x}_{\mathbf{0}}, \mathbf{x}_{\mathbf{f}} \in \mathbb{R}_{+}^{n}$ sebarang. Karena $\mathbf{x}_{\mathbf{f}} \in$ $\mathbb{R}_{+}^{n}$ maka $\mathbf{x}_{\mathbf{f}} \in \mathcal{R}_{\infty}(E, A, B)$ yang berarti bahwa $\mathbf{x}_{\mathbf{f}} \in \mathbb{R}_{k}(E, A, B)$ untuk suatu $k$, akibatnya ada $\mathbf{u}(j) \geq \mathbf{0}, j=$ $0,1,2, \ldots, k+q-1$ sedemikian sehingga $\mathbf{x}_{\mathbf{f}}$ dibangun oleh kolom-kolom matriks $\mathcal{R}_{k}(E, A, B)$

$=\left\langle E^{D} B, E^{D} E^{D} A B, \ldots, E^{D}\left(E^{D} A\right)^{k-1} B,-(I\right.$ $\left.\left.-E^{D} E\right) A^{D} B, \ldots,-\left(I-E^{D} E\right)\left(E A^{D}\right)^{q-1} A^{D} B\right\rangle$. Karena $E^{D} A$ adalah suatu matriks nilpoten dengan indeks nilpotensi $l$, pilih $k \in \mathbb{Z}_{+}$dengan $k \geq l$, maka akibatnya

$$
\begin{aligned}
\mathbf{x}_{\mathbf{f}}=\sum_{i=0}^{k-1} E^{D}\left(E^{D}\right)^{k-i-1} B \mathbf{u}(i)-(I \\
\left.-E E^{D}\right) \sum_{i=0}^{q-1}\left(E A^{D}\right)^{i} A^{D} B \mathbf{u}(k \\
+i) .
\end{aligned}
$$

Fakta 3.2 memperlihatkan bahwa keadaan $\mathbf{x}_{\mathbf{f}}$ tercapai disebabkan karena adanya $\mathbf{x}_{\mathbf{0}} \in \mathbb{R}_{+}^{n} \quad$ sehingga sistem diskrit $(E, A, B) \geq 0$ terkontrol.

\section{Kesimpulan}

Berdasarkan uraian dari hasil dan pembahasan, dapat diberikan beberapa kesimpulan sebagai berikut:

1. Suatu sistem diskrit $(E, A, B) \geq 0$ adalah tercapai jika dan hanya jika, $\mathcal{R}_{\infty}(E, A, B)=$ $\mathbb{R}_{+}^{n}$.

2. Sistem diskrit $(E, A, B) \geq 0$ dengan $E E^{D} \geq$ 0 dan $E A=A E$ adalah tercapai jika dan hanya jika, dan

$$
\mathcal{F}_{\infty}(E, A, B)=\operatorname{Im}\left(E E^{D}\right)
$$

$$
\mathcal{B}(E, A, B)=\operatorname{ker}\left(E E^{D}\right) .
$$

3. Sistem diskrit $(E, A, B) \geq 0$ dengan $E E^{D} \geq$ 0 dan $E A=A E$ adalah terkontrol jika dan hanya jika tercapai dan terkontrol null.

\section{Referensi}

[1] Anton, H. Aljabar Linier Elementer Edisi Kedelapan-Jilid 1. Erlangga. Jakarta (2004) 
[2] Arifin, A. Aljabar Linear Edisi Kedua, Penerbit ITB. Bandung (200)

[3] Bru, R., Coll, C., Sanchez, E. Structural Properties of Positive Linear TimeInvariant Difference-Algebraic Equations. Lin. Alg. Appl. Vol. 349 pp: 1-10 (2002)

[4] Campbell, S.L., Meyer. C.D. and J.R. Nicholas. Applications of the Drazin Invers to Linier Systems of Differential Equations with Singular Constant Coefficients. SIAM J. Appl. Math. Vol. 31 no. 3 pp: 411-425 (1979)

[5] Canto, B., Coll, C., Sanchez, E. Positive Solutions of a Discrete-Time Descriptor System. International Journal of Systems Science. Vol. 39 no. 1 pp: 81-88 (2008)

[6] Gantmacher, F.R. The Theory of Matrices. Vol 1. AMS Chelsea Publishing. Rhode Island (2000)

[7] Hoffman, K., Kunze, R. Linear Algebra Second Edition. New Jersey (2006)

[8] Jacob, Bill. Linear Algebra. W. H. Freeman and Company. New York (1990)

[9] Kaczorek. T. Linier Control Systems. Vol. 1. Research Studies Press LTD. England (1992)

[10] Noutsos, D., Tsatsomeros, M.J. Reachability and Holdability of Nonnegative States. SIAM Journal on Matrix Analysis and Applications. Vol. 30 pp: 700-712 (2008)

[11] Rahmalina, Widdya. Thesis, Sistem Deskriptor Diskrit Positif, Department of Mathematics, Faculty of Mathematics and Natural Sciences, Universitas Andalas, Padang, 2011

[12] Retno Sari, Yulia. Sistem Deskriptor Diskrit Linier Positif yang Terkontrol Null. Journal Proceeding Seminar Nasional Matematika STKIP PGRI. Padang, 2015

[13] Roman, S. Advance Linear Algebra. Springer. New York, (1992)

[14] Serre, Denis. Matrices Theory and Application. Second Edition. Springer. France (2010) 